\title{
The dynamical approach to spacetime theories
}

\author{
Harvey R. Brown* and James Read ${ }^{\dagger}$
}

April 25, 2018

Faculty of Philosophy, University of Oxford, Radcliffe Humanities, Woodstock Road, Oxford OX2 6GG, UK.

\begin{abstract}
We review the dynamical approach to spacetime theories - in particular, (a) its origins in the development of special relativity, (b) its opposition to the contemporary 'geometrical' approach, and (c) the manner in which it plays out in general relativity. In addition, we demonstrate that the approach is compatible with the 'angle bracket school'.
\end{abstract}

\section{Contents}

1 Introduction $\quad 2$

2 Maxwell's electrodynamics $\quad 3$

$\begin{array}{lll}3 & \text { Explanation } & 7\end{array}$

4 General relativity $\quad 13$

5 Coordinate-independent approaches $\quad 17$

6 Outlook 21

*harvey.brown@philosophy.ox.ac.uk

†james.read@philosophy.ox.ac.uk 


\section{Introduction}

In 1940, Einstein offered the following nutshell account of his special theory of relativity (SR): ${ }^{1}$

The content of the restricted relativity theory can ... be summarised in one sentence: all natural laws must be so conditioned that they are covariant with respect to Lorentz transformations.

[17, p. 329]

This innocuous-sounding statement actually represents on Einstein's part a significant departure from his 1905 'principle theory' approach to SR, based upon the relativity principle, the light postulate, and the isotropy of space. The shift in his thinking did not come about overnight. He had been disconcerted for many years by both the limitations of the thermodynamic template he had used in 1905 with its appeal to phenomenological principles, and the inordinate emphasis he had placed on the role of light - and therefore electromagnetism - in his theory. ${ }^{2}$

The so-called 'dynamical' approach to spacetime theories can be considered a development of Einstein's 1940 characterisation of SR, and an attempt to extend it both to general relativity (GR), and to a broader context of spacetime theories. We defer the details until later in this paper, but note in the meantime a feature of the 1940 statement common to the 1905 treatment: the absence of reference to the 'geometry of spacetime', and emphasis on the (non-gravitational) laws of nature.

The seeds of this dynamical approach were sown in the late 19th century by the great ether theorists G. F. FitzGerald, Hendrik Antoon Lorentz, Joseph Larmor, and Henri Poincaré. The earliest post-1905 articulations can be found in the writings of such luminaries as Hermann Weyl, Arthur Eddington, and Wolfgang Pauli, and it is not unreasonable to put Einstein himself into the mix. Later proponents included William F. G. Swann, Lajos Jánossy, John S. Bell and Dennis Dieks. ${ }^{3}$ Notable recent articulations

\footnotetext{
${ }^{1}$ If a dynamical equation retains the same form in either of two coordinate systems under consideration, then in this paper we say that it is invariant under the coordinate change relating those systems. This differs from both Einstein and [8], where the term covariant is used. The terminology of this paper is in line with [43]. In addition, where Einstein spoke of Lorentz covariance (for us: Lorentz invariance), we often speak in this paper of the broader notion of Poincaré invariance, for reasons which will become clear in $\S 5$.

${ }^{2}$ Einstein also had a bad conscience about the "sin" of treating ideal rods and clocks as primitive entities in his 1905 formulation. For discussion of these misgivings, see [8, $§ 7.1]$.

${ }^{3}$ For details concerning all these historical claims, see [8, chs. 4, 7].
} 
within the physics literature are due to D. J. Miller and William N. Nelson. ${ }^{4}$ In the last few decades, the approach has come to life within the philosophy of physics as a reaction to aspects of the 'angle bracket school' of spacetime theories, first prominently exposed in the philosophical literature of the 1970s and especially the 1980s. ${ }^{5}$ The central role of geometry in the treatment of pre-general relativistic theories in this approach has led some philosophers to the view that special relativistic effects such as length contraction and time dilation are ultimately explained by recourse to the geometric structure of Minkowski spacetime, and that all such explanations prior to the 1908 work of Minkowski are either misguided or incomplete. Such a view still has weighty defenders, as the paper by Maudlin in this volume attests. Defenders of the dynamical approach take issue with this view.

A review of this size cannot do justice to recent criticisms of the dynamical approach, but at least its conceptual grounding can be laid out. To this end, we begin with Maxwell's 1865 theory of electrodynamics: the first special relativistic theory in the history of physics.

\section{Maxwell's electrodynamics}

In saying that Maxwell's electrodynamics is (special) relativistic, we are referring to the standard field equations, possibly with source terms, but not in combination with the original (non-relativistic) Lorentz force law. ${ }^{6}$ Despite this theory's unique role as the historical cradle of SR, the latter theory transcends it, as Einstein would insist in his mature years. SR amounts to a specific constraint on all the non-gravitational interactions, not just the electromagnetic, and it transcends the exact form of the equations of these interactions when written in inertial coordinate systems. What SR demands is simply that the equations governing all these interactions be Poincaré invariant. It is satisfaction of this condition that makes Maxwell's field equations special relativistic. ${ }^{7}$

\footnotetext{
${ }^{4}$ See $[32,34,35]$.

${ }^{5}$ See in particular Michael Friedman's classic [18]. Note, however, that debates over the primacy of coordinate-independent approaches in the foundations of spacetime theories should in general be divorced conceptually from debates regarding the dynamical approach to spacetime- see $\S 3.3$.

${ }^{6}$ Strictly speaking, we are referring here to the formulation of the theory in terms of the 3 -vector formalism later developed by Heaviside and Hertz. It is only with the 4-tensor formulation of Minkowski that the theory becomes manifestly Poincaré invariant.

${ }^{7}$ In [8, pp. 146-147], this condition is referred to as the big principle.
} 
Looking forward to GR, Maxwell's name reappears in the context of the Einstein-Maxwell field equations. It is often thought that the fragment of these equations related to the electromagnetic field is 'locally' equivalent to the original nineteenth century theory. Although this is not entirely true for several reasons, this (more fundamental) general relativistic fragment is still locally Poincaré invariant. ${ }^{8}$ We shall return in $\S 4$ to discuss further such local dynamical equations. In the meantime, we wish to counter what we see as two common confusions concerning the Maxwell theory as understood prior to Einstein's revolution, as a warm-up to defending the dynamical approach to spacetime theories.

(i) The first is that the theory is incomplete. The theory, the argument goes, does not specify explicitly the spacetime structure required for the very definition of the technical terms it employs. As John Earman affirmed in 1989, "...laws of motion cannot be written on thin air alone but require the support of various space-time structures" [15, p. 46]. At first sight, this claim should strike one as puzzling. What physical effect do Maxwell's equations, in say 3-vector form, fail to predict, or leave ambiguous, that more explicit versions might not? None, if one bears in mind that in the nineteenth century inertial frames were taken to be global. (More on this later.) Recall John S. Bell's perceptive remark in his 1976 essay How to teach special relativity, that "the laws of physics in any one reference frame account for all physical phenomena, including the observations of moving observers" [4, p. 77].

It turns out that defenders of the incompleteness claim are likely, in our experience, to be those brought up in what is sometimes called the 'angle bracket school', according to which a spacetime theory first specifies a triple structure $\langle M, A, D\rangle$ (no pun intended!), where $M$ is the space-time manifold, $A$ is a placeholder for 'fixed' geometric-object fields characterising fixed spacetime structure (if any), ${ }^{9}$ and $D$ represents 'dynamical' geometric-object fields. The theory then has the job of specifying which of the kinematically possible models (KPMs) built out of this structure are dynamically allowed (i.e., are dynamically possible models (DPMs)), the elements of $A$ being unchanged in each kinematically (a fortiori dynamically) allowed model of the theory. Certainly, this geometrical approach has a number of merits, not the least of which is allowing for successive theories from Galileo to Newton

\footnotetext{
${ }^{8}$ See [43].

${ }^{9}$ In light of subtleties regarding the notion of an 'absolute object', and the question of whether GR itself has such objects (see [37]), in this paper we follow the nomenclature of [40] in referring to the objects picked out by $A$ as 'fixed fields'.
} 
to Maxwell to Einstein (SR and GR) to be formulated in a common formal language. ${ }^{10}$ (In the case of GR, there are no fixed fields $A$ in the usual sense, and $D$ stands for the metric field together with the energy-momentum tensor(s) associated with matter fields, if any.)

This unified language typically involves dynamical equations that are generally covariant-i.e., which are form-invariant under arbitrary coordinate transformations. ${ }^{11}$ The traditional Maxwell equations (even in the form provided by Minkowski) are not. Is this a defect? Here opinions are bound to differ. We see nothing intrinsically superior about general covariance; the key issue is which coordinate systems most simplify the form of the equations of the relevant dynamical theory. ${ }^{12}$ In the language of Friedman $[18$, p. 60$]$, in which coordinates is the "standard formulation" of the theory obtained? In the case of Maxwell theory, it is the inertial coordinates in which the Poincaré-Einstein synchrony convention is adopted, related by Poincaré transformations. The argument for general covariance which rests on the claim that coordinate systems are mere labels of space-time events, and that theories should be expressed in a label-general way, strikes us as naïve. How is it in SR, as understood by Einstein in 1905, that the Lorentz transformations - supposedly mere changes of labels - can encode the physical phenomena of length contraction and time dilation? (For further discussion of these matters, see a recent, enlightening study of the role of coordinate systems in physics by David Wallace [47], and $\S 5$ below.)

(ii) The second and related confusion about Maxwell's electrodynamics is that, prior to Einstein, the theory must be thought of as being defined in pre-relativistic spacetime. By this, we mean that at the very least, inertial coordinate transformations involving boosts are Galilean, not Lorentzian. Let's return again to Bell's 1976 essay, and its dynamical analysis of a hydrogen-like atom. Bell showed that if one chooses an inertial frame involving the Poincaré-Einstein convention, so that Maxwell's equations take their familiar form therein, the predictions concerning the shape and period

\footnotetext{
${ }^{10}$ This is achieved in unprecedented detail in [18]; see also [15, 30].

${ }^{11}$ In light of footnote 1 , it would be preferable to refer to this property as general invariance; this we do not do for historical reasons. The observation that any spacetime theory can be written in a generally covariant form was made to Einstein by Kretschmann in 1917 [27].

${ }^{12}$ In [6], the desideratum of general covariance in formulating theories like Maxwell's electrodynamics is compared with the desideratum of formulating globally gauge-invariant quantum dynamics in locally gauge invariant terms, when external fields (curvature) are absent. Earman [15, ch. 3, $\S \S 4-5]$ also warns that the 'fiddling' involved in expressing equations of motion in generally covariant form can lead to misunderstandings if the full significance of the original equations is not taken into account.
} 
of an inertially moving atom obtained by solving the equations in this frame will be consistent with those obtained by Lorentz transforming to the rest frame of the atom and using the standard form of Maxwell's equations in that frame. Admittedly, Bell's analysis used, over and above the Maxwell field equations, relativistic dynamics of the kind that features in the relativistic version of the Lorentz force law. But Galilean transformations, if they have anything like the operational significance we attribute to them today, are not easy to reconcile with Maxwell's field equations.

Here is another way to make this point. Consider the simple light- or Langevin-clock: two separated mirrors mounted in parallel on, and perpendicular to, a rigid straight rod. A pulse of light traveling parallel to the rod bounces back and forth between the mirrors. When at rest in the frame relative to which Maxwell's equations are valid, the period of the clock is approximately $2 L / c$ where $L$ is the distance between the mirrors, assumed to be large compared to the pulse width, and $c$ is the light speed as measured in that frame. Maxwell's equations predict that $c$ is constant, i.e. independent of the speed of the source and isotropic. It follows that if the Langevin clock is now boosted into a new state of inertial motion with speed $v$ relative to the original frame, and in the direction of the principal axis of the rod, the period becomes (as calculated by the observer in the original 'resting' frame) $2 \gamma^{2} L / c$, where $\gamma$ is the familiar Lorentz factor $\left(1-v^{2} / c^{2}\right)^{-1 / 2}$. It is assumed that the length of the rod is not affected by the boost, again as determined by the resting observer. Assuming more generally that the Galilean transformations hold, and have the standard operational meaning, it also follows that there can be no time dilation associated with moving clocks. When applied to the Langevin clock, this constraint implies that the proper period of the moving Langevin clock does not coincide with that in its resting state. ${ }^{13}$ But note that, as Einstein himself stressed, ${ }^{14}$ it is a fundamental condition for the very possibility of kinematics such as exemplified by the Galilean transformations, that ideal clocks are boostable, meaning that as long as the forces producing the boosts are weak enough, the proper periods of such clocks in their equilibrium states are invariant under the boosts. So the Langevin clock is a counterexample to this principle, and this conclusion should also shake our faith in the notion that Maxwell's equations can be combined with Galilean kinematics.

Such considerations are, however, entirely absent in attempts within the angle bracket school to formulate a generally covariant version of 'classical'

\footnotetext{
${ }^{13}$ See [8, p. 44].

${ }^{14}$ See [16] and [8, pp. 30, 81].
} 
Maxwellian electrodynamics within a space-time structure which purports to be consistent with the Galilean law of the transformation of velocitiesattempts to provide a rigorous systematisation of presumably what Maxwell himself thought was going on. ${ }^{15}$ Of course it is acknowledged that such theories are hard to reconcile with the null results of ether wind experiments, especially that of Michelson and Morley in 1887. But the issue here is not whether classical electrodynamics is false; it is whether it is coherent. What is lost sight of within the geometric intricacies of such attempts is the fact that physical rulers and clocks are complicated dynamical objects subject to the very non-gravitational interactions that are in play. In other words, what is overlooked is the fact that the distinction between kinematics and dynamics is not fundamental. ${ }^{16}$

Even the great Maxwell failed to see this. We expect undergraduates to imbibe in their first course on relativity theory a profound insight largely obscure to all the nineteenth century giants, including Maxwell, Lorentz, Larmor and Poincaré: the physical meaning of inertial coordinate transformations. It was Einstein in 1905 who was the first to understand the physics of such transformations, ${ }^{17}$ and the fact they are neither a priori nor conventional. This achievement in our view outstripped his treatment of length contraction, time dilation and relativity of simultaneity - effects that were, after all, already in the air at the turn of the century. ${ }^{18}$ Nor was this achievement based on a temporary state of philosophical derangement on Einstein's part associated with crude operationalism. No wonder he was unimpressed two decades later when Bohr and Heisenberg picked up what they thought was the Einstein banner and applied crude operationalism in their disturbance theory of indeterminacy in quantum theory.

\section{Explanation}

One of the most significant claims that the dynamical approach calls into question has to do with the nature of the explanation of the so-called kinematical relativistic effects of length contraction and time dilation. No one, to our knowledge, questions that these effects are a consequence of the Poincaré

\footnotetext{
${ }^{15}$ See [18, ch. III, §5].

${ }^{16}$ For a more detailed critique of 'classical electrodynamics', see [5]. Another notable feature of the angle bracket school (at least on the part of some of its prominent advocates) is the way in which the Newton-Einstein relativity principle is formulated. This has also been the subject of criticism; the interested reader may consult $[45,6,13]$, and [8, p. 36].

${ }^{17}$ Well, there was Keinstein in 1705, but that's another story. See [8, ch. 3].

${ }^{18}$ See [8, ch. 4].
} 
invariance of the laws governing the non-gravitational interactions that are responsible for the forces of cohesion within 'rigid' rulers and 'ideal' clocks. The bone of contention is whether the buck stops there.

\subsection{Symmetries, and the dynamical approach}

Consider again a spacetime theory specifying a triple $\langle M, A, D\rangle$, with fixed spacetime structure, and hence containing geometric-object fields associated with the entry $A$. Following Earman [15, p. 45], a spacetime symmetry is defined as a mapping from $M$ onto itself that preserves (leaves invariant) each element of $A$. In contrast, a dynamical symmetry is defined as a mapping from $M$ onto itself such that if $\langle M, A, D\rangle$ is a dynamically allowed model of the theory, then so is the model obtained by dragging along the elements of $D$ induced by the mapping. ${ }^{19}$ In the case of Minkowski spacetime, the spacetime symmetries are the isometries of the Minkowski metric; the dynamical symmetries are related to the Poincaré group of coordinate transformations which preserve the form of the equations of motion when written in inertial coordinates. ${ }^{20}$

In his famous discussion in 1989, Earman claimed that that every spacetime symmetry is a dynamical symmetry, and vice versa $[15$, ch. $3, \S 4]$. He stressed that this double requirement is not a matter of definition, but represents "adequacy conditions" for the theory in question to be "well-tuned". Now it is well-known that Newtonian mechanics set in 'Newtonian' spacetime has dynamical symmetries that are not spacetime symmetries. Earman argued that Newtonian spacetime should be replaced by 'neo-Newtonian' (sometimes: 'Galilean') spacetime, essentially on the grounds of Occam's razor: the former has absolute structure which is in an important sense idle.

\footnotetext{
${ }^{19}$ Earman defines both spacetime and dynamical symmetries in terms of diffeomorphisms; this restriction excludes discrete symmetries associated with time reversal and space reflection (parity). In our view, it is not clear on what grounds symmetries involving discrete rather than continuous (Lie) groups are so excluded. Cf. $\S 3.2$.

${ }^{20}$ Note that, if the notion of spacetime symmetries is cashed out in terms of the transformation properties of fixed fields $A$, then the above analysis cannot be applied to e.g. GR, or the alternative formulation of SR presented at [40, p. 120], for such theories feature no fixed fields. A change of nomenclature broadly suffices to solve this difficulty - see [39, $\S 3.1]$, according to which 'spacetime symmetry groups' are "groups of transformations that preserve spatiotemporal structure", and a 'dynamical symmetry group' "is a group that preserves the form of the equations that express the dynamical laws." Note, however, that this particular choice of nomenclature presupposes that the geometrical objects associated with 'spacetime' may be picked out from the triple structure associated with a given theory; such an assumption is questionable on the dynamical approach (see [8, ch. 9]).
} 
As for whether a spacetime symmetry may not be a dynamical symmetry, Earman found it hard to envisage such a possibility.

There is a sense in which the advocate of the dynamical approach can agree with this latter verdict of Earman. However, there is also a sense in which, for the advocate of the dynamical approach, the former scenarioviz., cases in which dynamical symmetries outstrip spacetime symmetriesis equally difficult to envisage. The reason for this is that, as discussed elegantly by Myrvold in a recent paper [33], the advocate of the dynamical approach regards the coincidence of spacetime and dynamical symmetries not merely as an "adequacy condition" on a given theory, but rather indeed as holding analytically, "in virtue of considerations of meaning" [33, p. 7]. By proceeding in this manner, any mystery regarding how spacetime structure is supposed to 'explain' the dynamics of matter is dissolved.

Myrvold tells us that spacetime structure just is that fixed structure $A$ the spacetime symmetries of which coincide with the dynamical symmetries of the theory in question. Though we broadly concur with this presentation, it is possible that Myrvold's account might give the impression that the advocate of the dynamical approach is interested principally in conceptual analysis - spacetime symmetries just are dynamical symmetries; spacetime structure (ultimately reducible to symmetries of dynamical laws) just is that structure which manifests those symmetries. ${ }^{21}$ It is important to note, however, that advocates of the dynamical approach may resist being thus tarred with the analytical brush. Rather, the principal aim of this approach is to account for the chronogeometricity of metric structure - that is, to answer the question of why that structure is surveyed by rods and clocks built out of matter fields; this is achieved by stating that the metric field in such theories is reducible to dynamical symmetries. There is a sense in which the term 'spacetime' is redundant to this goal; we anticipate that some advocates of the dynamical approach would be happy to excise it. ${ }^{22}$

\subsection{The geometrical approach}

Having sketched some key aspects of the dynamical view, let us turn now to a diametrically opposite approach. It is sometimes argued that the symmetries of Minkowski spacetime explain the fact that the dynamical laws

\footnotetext{
${ }^{21}$ Cf. [26, p. 2].

${ }^{22}$ When viewed in this manner, the spacetime functionalism of Knox can be understood as augmenting the dynamical approach, essentially with the functionalist criterion that 'spacetime' should be identified with whatever structure in a given theory has chronogeometric significance. For more on Knox's approach, see [24, 25, 26].
} 
are Poincaré invariant. Such a view is implicit in Friedman's 1983 book. $^{23}$ Though not himself not an advocate of the angle bracket school generally, Michel Janssen wrote similarly in 2002:

In Minkowski spacetime, the spatio-temporal coordinates of different observers are related by Lorentz transformations rather than Galilean transformations. Any laws for systems in Minkowski spacetime must accordingly be Lorentz invariant. [22, p. 499]

More recently, Tim Maudlin has likewise stated:

... the Minkowski geometry takes exactly the same form described in [any] Lorentz coordinate system (by the symmetry of Minkowski spacetime), and the laws of physics take exactly the same coordinate-based form when stated in a coordinate-based language in any Lorentz coordinate system (because the laws can only advert to the Minkowski geometry, and it has the same coordinate-based description). [31, pp. 117-118]

Readers may recall Bell's 1976 discussion of a string connecting two rockets undergoing identical accelerations. Maudlin argues that the length contraction responsible for the eventual breaking of the string can be traced to three circumstances:

... the geometrical symmetries in Minkowski spacetime, the necessity of specifying dynamical laws in terms of the Minkowski structure, and the physical constitution that makes the thread a rigid body ... [31, p. 119]

It is somewhat ironic that Bell was able (as Einstein would have been in 1905, but with different emphasis) to explain the string breaking effect without ever referring to Minkowski spacetime; indeed one of the aims of Bell's increasingly well-known paper was to warn against "premature philosophising about space and time" $[4$, p. 80$]$ when teaching special relativity. Was he misguided?

Fixed geometric structures in physics are not limited to spacetime. Consider, for example, the Lobachevskian geometry of the velocity space in special relativity that is associated with the non-commutativity of (nonaligned) boosts and hence the Thomas precession effect, or the curved metric of the projective Hilbert space in quantum mechanics associated with

${ }^{23}$ See e.g. [18, ch. VI, §4]. 
the phenomenon of geometric phase. ${ }^{24}$ These different geometric structures (discovered after the physical effects to which they are associated) are rather elegant mathematical representations of aspects of the physics of boosts and of Schrödinger evolution, respectively, but arguably do not constitute an $e x$ planation of these effects. Why should it be any different in the case of absolute spacetime geometry?

Then there is the question of how the explanation suggested by Janssen, Maudlin, and others, is supposed to work. Does Minkowski spacetime somehow 'act' on the dynamical geometric-object fields? Friedman made it clear in 1983 that Minkowski spacetime is in his view a real physical entity. ${ }^{25}$ Is it then capable of action? It is certainly not capable of reaction, because it is a fixed structure. If it acts in some causal way, it is a curious business: no terms relating to the action appear in the dynamical laws when expressed in their standard configuration. ${ }^{26}$ (Hence the possibility of a relativistic theory - Maxwell's electrodynamics - appearing decades before spacetime was a glint in either Poincaré's or, more importantly, Minkowski's eyes.) The use of vague expressions like the necessity of laws to 'advert' to the Minkowski geometry does little to dispel the mystery.

By way of development on this point, consider the standard model of particle physics (following [13, p. 256]). In this model, the strong and electromagnetic force are known to conserve parity (i.e. the associated laws are invariant under the discrete symmetry of spatial reflections); not so for the weak force, which violates parity and time-reversal invariance. In order to 'explain' weak force dynamical laws, advocates of the geometrical approach presumably must introduce extra spacetime structure, over and above the Minkowski metric, in order to ensure that spacetime and dynamical symmetries in the sense of Earman align. ${ }^{27}$ The extra structure involved in the case of the weak interactions would be a continuous choice throughout the manifold $M$ determining which half of the null cone represents the future,

\footnotetext{
${ }^{24}$ For more details, see [8, pp. 134-136].

${ }^{25}$ See $[18$, ch. VI, §4]. Note, however, that Janssen does not embrace such a claim, speaking instead of a 'common origin inference' to Minkowski spacetime structure, understood as universal Lorentz invariance construed as a kinematical constraint. See e.g. [22, 23], and discussion below.

${ }^{26}$ It is worth noting that a few years after proposing his gravitational field equations, Einstein criticized both Newtonian mechanics and SR for violating the action-reaction principle, because the relevant spacetime structures in both cases allegedly determined the inertial motion of free bodies. We return to the issue of inertia below. For a recent critical discussion of Einstein's stance and its timing, see [9].

${ }^{27}$ At least, if such discrete symmetries are also subject to Earman's "adequacy conditions" - cf. footnote 19.
} 
and an antisymmetric tensor field which similarly provides a continuous choice of 'right handed' versus 'left handed' orthonormal triads of spacelike vectors at each point in $M .{ }^{28}$ But, having introduced this structure, the question arises: why does the strong force, for instance, not also 'advert' to this spacetime structure? Some further nuancing of the notion of geometric explanation is surely needed here. ${ }^{29}$

Another awkwardness in the geometric approach is exposed in the case of the de Broglie-Bohm hidden-variable quantum theory. In its relativistic treatment of quantum fields, the equation governing the quantum degrees of freedom is Poincaré invariant; however, the guidance equation governing the sub-quantum degrees of freedom is Galilean invariant, and in general involves instantaneous action-at-a-distance. From the geometric perspective it seems that the theory invokes two distinct spacetime structures-Minkowski and Galilean ${ }^{30}$ - though the latter is 'hidden'. ${ }^{31}$ It is unclear how the geometric explanation of dynamical symmetries is supposed to work in this case. ${ }^{32}$ To reject the theory on the grounds that its spacetime structure is ambiguous, or degenerate, would surely be too high-handed!

It is well-known that Newton attributed no powers of action to absolute space, not even to constrain the inertial motion of force-free bodies. It is noteworthy that in attempting to account for the universality of Poincaré invariance in terms of Minkowski spacetime, Janssen accepted in 2009 that this geometric structure has no existence independent of the matter fields subject to the dynamical laws of interest, so its action is not causal [23, p. 28]. Janssen gives a number of overlapping accounts as to what it is, one being

\footnotetext{
${ }^{28}$ See [46, p. 60] and [20]. Note that it is not just the weak interactions that seem to call for this augmented Minkowski spacetime. In local relativistic QFT, it is a theorem that for systems of identical particles confined to two spatial dimensions, if either spatial reflection in a line or time reversal is a symmetry of the theory in all its superselection sectors, then the quantum statistics are associated with the ordinary permutation group, i.e. either Bose-Einstein or Femi-Dirac. See [19, p. 56]. However, remarkably, the possibility of 'fractional' or 'braid' statistics ranging between Bose-Einstein and Fermi-Dirac is known to be self-consistent in the case of two-dimensional confinement, in a way that it is not when electrons explore three dimensions. See [29]. This phenomenon is not a mere theoretical nicety - it is apparently displayed in two-dimensional electron gases in a transversal external magnetic field exhibiting the fractional quantum Hall effect (see e.g. [41]). Space reflections and time reversal are not symmetries of such electron gases [19, p. 56]. Certain systems exhibiting the fractional quantum Hall effect are being investigated with a view to application in quantum computation.

${ }^{29}$ For further recent discussion on this point, see [42].

${ }^{30}$ See e.g. [30, ch. 4] for technical details.

${ }^{31}$ For further details see [36] and [44].

${ }^{32}$ For related comments, see [33].
} 
a kind of nomic necessity inspired by Marc Lange's analysis of 'meta-laws' in physics [28]. Rather, however, than pursue this line of thinking, we wish to make two points. The first is that there is nothing in Minkowski's own writings suggesting that he was providing an explanation for the universal nature of the Poincaré group; on the contrary, it was the latter on which he based his geometrical insights. ${ }^{33}$ Second, the dynamical approach outlined in $§ 3.1$ constitutes a straightforward means of resolving these mysteries.

\subsection{The dynamical approach, reprise}

It should be clear that the dynamical approach to spacetime theories is to be set in contrast with the above geometrical account. One further aspect of the dynamical approach is now worth emphasising: in the context of SR, Poincaré invariance of the dynamical laws is, on this view, to be understood as a brute fact: no 'explanation' of such a result via appeal to spacetime structure is mandated; nor is it clear how such an account could proceed. In a theory in which all dynamical equations for non-gravitational fields manifest the same symmetries - again, such as SR - spacetime structure may be viewed as a codification of such symmetries.

Having thus presented the dynamical approach, it is worth dispelling one possible source of confusion. While the geometrical approach is often endorsed by members of the angle bracket school (such as Friedman [18, ch. IV]), and advocates of the dynamical approach (such as ourselves) often do not view as illegitimate the issuing of coordinate-dependent presentations of physical theories ( $\mathrm{cf} . \S 2$ ), the debate between coordinate-dependent versus -independent presentations should be considered orthogonal to the debate between the geometrical and dynamical approaches. This can be illustrated through two points: on the one hand, authors such as Janssen can be viewed as embracing (a certain version of) the geometrical approach alongside coordinate-dependent presentations; on the other, we shall see in $\S 5$ that there may be some advantages to viewing the dynamical account through the lens of coordinate-independent approaches to spacetime theories.

\section{General relativity}

In the context of theories with non-dynamical metric fields such as SR, one crucial aspect of the debate between the dynamical and geometrical

\footnotetext{
${ }^{33}$ See [8, ch. 8].
} 
perspectives may be put straightforwardly: for the advocate of the former, the metric field (in the case of SR, the Minkowski metric field) need not be regarded as an ontologically distinct and primitive entity; rather, it is a codification of certain brute facts about the dynamical laws governing matter fields (namely, facts about their symmetries). By contrast, for certain advocates of the latter, ${ }^{34}$ the metric field in such theories is an ontologically distinct and primitive entity, its presence explaining certain facts about the dynamical laws governing matter fields (namely, the fact that they all manifest certain symmetries).

This situation changes in important ways on moving to theories with dynamical metric fields, such as GR. Since in such cases the metric field comes equipped with its own associated field equations (in the context of GR, the Einstein field equations), this field is not straightforwardly ontologically reducible to the matter fields, as per the dynamical approach in the context of spacetime theories with non-dynamical metric fields. ${ }^{35}$ Indeed, advocates of both the dynamical and geometrical perspectives in the context of theories with dynamical metric fields are in agreement on this point.

That said, there remain crucial differences between the two camps, in particular regarding the chronogeometric significance of the metric field. On the dynamical perspective, the dynamics of the metric field tell us that it is 'just another field': "Nothing in the form of the equations per se indicates that [the metric field] is the metric of space-time, rather than a $(0,2)$ symmetric tensor which is assumed to be non-singular" [8, p. 160]. How, then, does the metric field acquire its chronogeometric significance in GR? For the proponent of the dynamical approach, the metric field "earns its spurs by way of the strong equivalence principle" [8, p. 151]. That is to say, locally in GR - assuming that one's experimental apparata are sufficiently insensitive that curvature effects may be ignored - not only does the metric field take the form of the Minkowski metric field (i.e. is diagonalisable, and invariant under Poincaré transformations), but dynamical equations for matter fields take a Poincaré invariant form. ${ }^{36}$ As a consequence, rods and clocks built out of matter fields locally survey the metric field. ${ }^{37}$

\footnotetext{
${ }^{34}$ Here we exclude Janssen-cf. footnote 25.

${ }^{35}$ To claim that the metric field is reducible to the matter fields in GR is to endorse a certain form of relationism about the metric field; there are profound difficulties with implementing this programme in GR. An obvious illustration of this difficulty can be found in the existence of vacuum solutions in the theory.

${ }^{36}$ These are significant subtleties in this vicinity - see [43, $\left.\S 33-4\right]$ for recent discussion.

${ }^{37}$ The above is posed in the context of GR, but if an equivalent form of the strong equivalence principle is available in other spacetime theories with dynamical metric fields, then the same analysis may go through.
} 
By contrast, advocates of the geometrical approach in the context of spacetime theories with dynamical metric fields may maintain that the metric field has a primitive connection to spacetime geometry - and that in the local regime in which curvature effects may be ignored, the dynamical laws governing matter fields are locally constrained to be invariant with respect to the local symmetries of this field, in the same manner as for the geometrical picture in the context of theories with non-dynamical metric fields, such as SR.

\subsection{Problem cases for the geometrical approach}

In this section, we present what we take to be two problem cases for the geometrical approach in the context of spacetime theories with dynamical metric fields. To begin, consider the Jacobson-Mattingly theory (presented in e.g. $\left.[14,21]^{38}\right)$, in which the action for a coupled Einstein-Maxwell system is augmented with an additional term (via a Lagrange multiplier field $\lambda$ ), imposing (as a field equation, via variation with respect to $\lambda$ ) that the vector potential $A^{a}$ be locally timelike: ${ }^{39}$

$$
S_{\mathrm{JM}}\left[g_{a b}, A^{a}, \lambda\right]=\int d^{4} x \sqrt{-g}\left(R-\frac{1}{4} F^{a b} F_{a b}+\lambda\left(g_{a b} A^{a} A^{b}-1\right)\right) .
$$

The imposition of this Lagrange multiplier term means that, in the Jacobson-Mattingly theory, the dynamical behaviour of non-gravitational fields does not reflect the local (Poincaré) symmetries of the metric field. Given this, however, we appear to have in our possession a problem case for the geometrical approach.

Turn now to our second case: Bekenstein's bimetric TeVeS ('TensorVector-Scalar') theory, presented in [2,3]. As discussed in [8, §9.5.2], in this theory the metric field which is surveyed by rods and clocks, the conformal structure of which is traced by light rays, and the geodesics of which correspond to the motion of free bodies, is not the 'fundamental' metric field $g_{a b}$, but rather a less 'fundamental' metric field $\tilde{g}_{a b}$, constructed from the other matter fields in the theory [8, p. 174]. Indeed, the $\mathrm{TeV} e \mathrm{~S}$ theory presents another case in which the local symmetries of the dynamical laws do not

\footnotetext{
${ }^{38}$ In fact, the version of the Jacobson-Mattingly theory discussed in this paper is a special case of that presented in [14, 21].

${ }^{39}$ The first term is the Einstein-Hilbert action; $F_{a b}$ is the Faraday tensor associated to $A^{a}$.
} 
mirror the local (Poincaré) symmetries of the 'background' metric field - a necessary condition for the geometrical approach to go through. ${ }^{40}$

\subsection{Two miracles}

Seemingly, the advocate of the dynamical perspective in the context of GR is committed to the existence of two unexplained input assumptions in the foundations of the theory-recently dubbed the two 'miracles of GR' [43, $\S 5]:{ }^{41}$

1. All non-gravitational interactions are locally governed by Poincaré invariant dynamical laws.

2. The Poincaré symmetries of the laws governing non-gravitational fields in the neighbourhood of any point coincide - in the regime in which curvature can be ignored - with the symmetries of the dynamical metric field in that neighbourhood.

(1) holds in SR: it tells us that the dynamical laws governing all matter fields are Poincaré invariant; the advocate of the dynamical approach takes this to be a brute fact; the advocate of the geometrical alternative attempts to rationalise this by appeal to Minkowski spacetime. (1) still obtains in the neighbourhood of any point in the spacetime manifold in GR. ${ }^{42}$ However, in the case of GR there also exists an ontologically autonomous metric field, and this leads to (2): why is it - assuming that curvature terms can be ignored (which, to repeat, depends upon the sensitivity of one's experimental apparata relative to the strength of curvature effects in the neighbourhood of that apparata) - that the symmetries of the dynamical laws governing non-gravitational fields in a suitable neighbourhood of any $p \in M$ coincide with those of the dynamical metric field in that neighbourhood? Again, the advocate of the dynamical approach may postulate this as a brute fact. By contrast, the advocate of the geometrical approach may attempt to argue that the ontologically primitive metric field explains the form of the dynamical laws governing matter fields; however, as in SR, she faces an outstanding burden to delineate how this is supposed to work. For further discussion regarding these 'miracles of GR', see [43, §5].

\footnotetext{
${ }^{40}$ For further detailed discussion of bimetric theories in defence of the dynamical approach, see [38]. Pitts also cites massive scalar gravity as a case in which "the chronogeometrically observable ... metric isn't clearly the One True Geometry" [38, §5].

${ }^{41}$ A similar 'miracle' in the context of Newtonian theory would be the proportionality of gravitational and inertial masses - cf. [48].

${ }^{42}$ See $[43, \S 3]$.
} 


\section{Coordinate-independent approaches}

Let us turn now in greater detail to a question raised in $\S 3.3$ : to what extent is the dynamical perspective reconcilable with the coordinate-independent, 'angle brackets' approach in the foundations of spacetime theories? Though this question has been addressed to some extent in $[39, \S 6.3 .2]$ and $[47, \S 6]$, it is worth reflecting further on the matter. In this section, we first consider theories with non-dynamical metric fields ( $\$ 5.1)$, before turning to theories with dynamical metric fields $(\S 5.2)$.

\subsection{Non-dynamical metric fields}

Consider the theory of a massive Klein-Gordon field $\varphi$, as specified in the coordinate-dependent approach via

$$
\eta_{\mu \nu} \partial^{\mu} \partial^{\nu} \varphi+m \varphi=0 .
$$

In (2), $\eta_{\mu \nu}$ denotes the components of a matrix diag $(-1,1,1,1)$. The choice of coordinate basis used in the above is (partly) arbitrary: this is captured in the fact that (2) is invariant under all Poincaré transformations, i.e. coordinate transformations of the form $x^{\mu} \rightarrow \Lambda_{\nu}^{\mu} x^{\mu}+a^{\mu}$, with $\Lambda^{\mu}{ }_{\nu} \Lambda^{\lambda}{ }_{\sigma} \eta_{\mu \lambda}=\eta_{\nu \sigma}$ and the $a^{\mu}$ arbitrary constant vectors. As elaborated in detail by Wallace [47, $\S \S 2-3]$, on the so-called Kleinian conception of geometry, dynamical equations such as (2) may be understood as defining certain spacetime structure, via their transformation properties - so, for example, (2) defines a Minkowski spacetime structure via the fact that it is invariant under Poincaré transformations. ${ }^{43}$ Clearly, this approach goes hand-in-hand with the dynamical perspective - spacetime structure may be read off from the transformation properties of the set of dynamical equations under consideration. ${ }^{44}$

\footnotetext{
${ }^{43}$ As Wallace states [47, p. 13], this approach is, in fact, also coordinate-independentfor it does not rely on evaluations being made in any particular coordinate system. For this reason, it may be preferable to call what in this paper we label the 'coordinateindependent approach' by some other name (though, as Wallace points out, the natural choice of 'coordinate-free' faces its own problems [47, p. 14]). For simplicity, however, we avoid doing so; this note should suffice to ameliorate any confusion in this regard.

${ }^{44}$ As Wallace states, "This is not to say that Brown's approach is compulsory in a presentation of spacetime theories based on structure (pseudo-)groups [roughly, groups of transformations which preserve dynamical equations in a given form]. I characterised such theories as having structure groups tacitly determined by the equations of motion, but it's mathematically coherent to start with a structured space with group $\mathcal{G}$ and then write down on that space a theory whose dynamical symmetry group is larger than $\mathcal{G}$ " $[47$, p. 22]. We take it that this latter deployment of the Kleinian conception of geometry would constitute a way of making sense of e.g. Janssen's position.
} 
The above in mind, suppose now that one seeks to reconcile the dynamical perspective in the context of such theories with the coordinateindependent approach. In the coordinate-independent picture, the theory in question is expressed through the introduction of the fixed geometrical object $\eta_{a b}$-a Minkowski metric field fixed identically in all KPMs of the theory; DPMs are then picked out by

$$
\eta_{a b} \nabla^{a} \nabla^{b} \varphi+m \varphi=0 .
$$

Since, however, this object is introduced in order to capture (in a coordinateindependent way) that the form of (2) is preserved only under Poincaré transformations ${ }^{45}$ we may understand this object $\eta_{a b}$ to codify the symmetry properties of (2) - this equation being understood to be fundamental, on the dynamical approach.

In this way, we understand fixed metric fields such as $\eta_{a b}$ to be ontologically supervenient upon the (dynamics of) matter fields (in this case $\varphi$ ); in doing so, we reconcile the coordinate-independent approach with the dynamical perspective. On this account, although the structure $\eta_{a b}$ appears in the KPMs of the theory in question, it is not to be understood as ontologically autonomous. Of course, the picture of introducing KPMs then DPMs remains highly unnatural on the dynamical view. Nevertheless, there is nothing erroneous in introducing these classes of models post hoc, while continuing to reconcile the primacy of coordinate-dependent dynamical laws for matter fields. ${ }^{46}$

\subsection{Dynamical metric fields}

Consider a minimally-coupled, ${ }^{47}$ general relativistic theory of the massive scalar field, analogous (as far as possible) to the special relativistic theory presented above. In the coordinate-dependent approach, such a theory may

\footnotetext{
${ }^{45}$ Since $\eta_{a b}$ is a fixed field, performing an (active) diffeomorphism implementing an arbitrary linear transformation on (3) yields $\eta_{a b} M^{a}{ }_{c} M^{b}{ }_{d} \nabla^{c} \nabla^{d} \varphi+m \varphi=0$, where $M^{a}{ }_{c}$ is the associated (linear) transformation matrix - and this will only reduce to (3) when $\eta_{a b} M_{c}^{a} M_{d}^{b}=\eta_{c d}$, i.e. when the linear transformation is a Poincaré transformation.

${ }^{46}$ Indeed, there exists a precedent in the angle brackets school of including nonfundamental geometrical objects in the KPMs of a given theory, for convenience - consider e.g. the inclusion of a derivative operator $\nabla_{a}$ over and above a timelike vector field $\sigma^{a}$ in the KPMs of Newtonian mechanics set in Newtonian spacetime. Our thanks to Tushar Menon for this point.

${ }^{47}$ For the definition of minimal coupling, as well as detailed philosophical discussion of the procedure, see $[12, \S \mathrm{IV}]$ and $[43, \S 3]$.
} 
be specified through writing down the following two dynamical equations: ${ }^{48}$

$$
\begin{aligned}
G_{\mu \nu} & =8 \pi T_{\mu \nu}, \\
g_{\mu \nu} \nabla^{\mu} \nabla^{\nu} \varphi+m \varphi & =0 .
\end{aligned}
$$

Here, $G_{\mu \nu}$ is the Einstein tensor associated to the metric field $g_{\mu \nu} ; T_{\mu \nu}$ is the stress-energy tensor associated to $\varphi$. Since in this case the metric field is dynamical, we have a theory for $g_{\mu \nu}$ and $\varphi$ together. ${ }^{49}$ The facts that (a) $g_{\mu \nu}$ is locally diagonalisable to read $\operatorname{diag}(-1,1,1,1)$; (b) locally and in a regime in which curvature effects may be ignored (this depends crucially upon the sensitivity of the experimental apparatus available relative to the strength of curvature effects - see $[43, \S 4]) g_{\mu \nu}$ has higher derivatives vanishing; and (c) in this regime (5) takes a Poincaré-invariant form, mean that $g_{\mu \nu}$ is locally surveyed by rods and clocks built out of the $\varphi$ field - thereby affording it its chronogeometric significance.

On the coordinate-independent approach, one characterises this theory as follows. First, KPMs are picked out by triples $\left\langle M, g_{a b}, \varphi\right\rangle$; then, DPMs are those KPMs the geometrical objects of which obey the dynamical equations

$$
\begin{aligned}
G_{a b} & =8 \pi T_{a b}, \\
g_{a b} \nabla^{a} \nabla^{b} \varphi+m \varphi & =0 .
\end{aligned}
$$

So be it-but ask now how one would express the dynamical perspective in coordinate-independent language in this case. Again, equations written in a coordinate basis - here (4) and (5) - are to be considered primitive. In this case, however, on moving to the coordinate-independent formalism, no new geometrical objects are introduced. ${ }^{50}$ This makes clear an important point already noted in $\S 4$ : in this case, metric field structure is not merely a codification of the symmetries of the given dynamical laws-rather, such structure already exists in (4) and (5).

Thus, consideration of the coordinate-independent formalism arguably illustrates a point in the context of spacetime theories such as GR to which the advocate of the dynamical approach assents. Perhaps more importantly, though, further merits of deploying this formalism emerge when one considers the local forms of (4) and (5). Considering (5) locally and in a regime in

\footnotetext{
${ }^{48}$ We set $G_{N}=c=1$.

${ }^{49}$ Cf. [40, p. 115].

${ }^{50}$ The reason for this is part of the special character of GR: (4) and (5) were generally covariant (i.e. held in an arbitrary coordinate basis) to start off with.
} 
which curvature terms may be ignored, this equation takes a form invariant under Poincaré transformations. ${ }^{51}$ Thus, applying the Kleinian conception of geometry to this new, local equation, one again extracts (in exactly the same manner as in §5.1) Minkowski spacetime structure - and again, on the dynamical approach, this structure can be understood as a codification of the symmetry properties of that local dynamical law.

Locally, therefore, one might introduce a Minkowski metic field $\tilde{\eta}_{a b}$ to codify the symmetries of this dynamical law. But note that the dynamical $g_{a b}$ field remains in this theory! Moreover, locally (and in a regime in which curvature effects can be dropped), $g_{a b}$ also resembles the Minkowski metric field. ${ }^{52}$ The test of whether $g_{a b}$ has local chronogeometric significance, therefore, is precisely whether the dynamical metric field $g_{a b}$ is locally isomorphic - in the appropriate regime in which curvature effects may be ignored - to the metric field which codifies the symmetries of the dynamical laws, $\tilde{\eta}_{a b}$.

Putting things in this way-i.e. introducing a new geometrical object taken to codify the symmetries of the original (non-gravitational) dynamical laws - is deeply ingrained in the coordinate-independent approach; nevertheless, it makes perspicuous the tests of chronogeometric significance envisaged by advocates of the dynamical approach in the cases in which the metric field itself is a dynamical entity. We conclude, therefore, that not only is the dynamical approach compatible with the coordinate-independent approach in this context; but moreover, there may be some advantages to its use, from the point of view of presenting the dynamical approach. ${ }^{53}$

Let us attempt to demonstrate this stronger claim via two auxiliary points. First, consider again bimetric theories with two dynamical metric fields. Using the above formalism, the metric field in these theories which may lay claim to chronogeometric significance is precisely that which is locally isomorphic to the metric field $\tilde{g}_{a b}$ which codifies the local symmetries of the dynamical laws. Second, thinking in this manner constitutes a good framework for consideration of scenarios in which there exists a misalignment between metric field structure and symmetries of the dynamical laws - for in such cases one may say that the dynamical metric field is not locally isomorphic to the metric field which codifies the symmetries of the non-

\footnotetext{
${ }^{51}$ This is demonstrated in e.g. [43, $\left.\S \mathrm{A}\right]$.

${ }^{52}$ Cf. [43, §3].

${ }^{53}$ Again, on the dynamical approach it is unnatural to introduce a class of KPMs in a coordinate-independent manner, then restrict to a certain class of DPMs based upon the dynamical equations which the geometrical objects in the KPMs satisfy. Nevertheless, there is nothing inconsistent with making use of this apparatus on the dynamical approach.
} 
gravitational laws.

\section{Outlook}

We have sketched the progression of the dynamical approach, from its origins in the development of SR, to its opposition to the contemporary 'geometrical' approach, to the manner in which it plays out in GR. Moreover, we have demonstrated that the perspective is not incompatible with certain coordinate-independent approaches in the foundations of spacetime theories. It is gratifying that work continues to be done in further developing the dynamical approach (see e.g. [24, 25, 26, 33, 38, 47, 49]); it is our hope that

its framework continues to offer insight to those working in the foundations of spacetime theories in years to come.

\section{Acknowledgments}

We are indebted to Eleanor Knox and Alastair Wilson for their invitation to write this review; and to Dennis Lehmkuhl, Tushar Menon, Simon Saunders, David Wallace, and especially Oliver Pooley, for many hours of invaluable discussions on the dynamical approach. J.R. is supported by an AHRC studentship, and is also grateful to Hertford College, Oxford, for a graduate senior scholarship.

\section{References}

[1] Yuri Balashov and Michel Janssen, "Presentism and Relativity", British Journal for the Philosophy of Science 54(2), pp. 327-346, 2003.

[2] Jacob D. Bekenstein, "An Alternative to the Dark Matter Paradigm: Relativistic MOND Gravitation, invited talk at the 28th Johns Hopkins Workshop on Current Problems in Particle Theory, June 2004, Johns Hopkins University, Baltimore. Available at arXiv:astro$\mathrm{ph} / 0412652$.

[3] Jacob D. Bekenstein, "Relativistic Gravitation Theory for the MOND Paradigm, available at arXiv:astro-ph/0403694.

[4] John S. Bell, "How to Teach Special Relativity", in Speakable and Unspeakable in Quantum Mechanics, second edition, Cambridge: Cambridge University Press, 2004. 
[5] Harvey R. Brown, "Correspondence, Invariance and Heuristics in the Emergence of Special Relativity", in S. French and H. Kamminga, (eds.), Correspondence, Invariance and Heuristics: Essays in Honour of Heinz Post, Dordrecht: Kluwer Academic Press, pp. 227-260,1993.

[6] Harvey R. Brown, "Aspects of Objectivity in Quantum Mechanics", in J. Butterfield and C. Pagonis (eds.), From Physics to Philosophy, Cambridge: Cambridge University Press, pp. 45-70, 1993.

[7] Harvey R. Brown, "The Origins of Length Contraction: The FitzGeraldLorentz Deformation Hypothesis", American Journal of Physics 69, pp. 1044-54, 2001.

[8] Harvey R. Brown, Physical Relativity: Spacetime Structure from a Dynamical Perspective, Oxford: Oxford University Press, 2005. Corrections were made in the 2007 (paperback) edition.

[9] Harvey R. Brown and Dennis Lehmkuhl, "Einstein, the Reality of Space, and the Action-Reaction Principle", in Partha Ghose (ed.), Einstein, Tagore and the Nature of Reality, London and New York: Routledge, pp. 9-36, 2016.

[10] Harvey R. Brown and Oliver Pooley, "The Origins of the Spacetime Metric: Bell's Lorentzian Pedagogy and its Significance in General Relativity", in C. Callender and N. Huggett (eds.), Physics Meets Philosophy at the Plank Scale, Cambridge: Cambridge University Press, pp. 256-72, 2001.

[11] Harvey R. Brown and Oliver Pooley, "Minkowski Space-time: A Glorious Non-entity", in Dennis Dieks (ed.), The Ontology of Spacetime, Amsterdam: Elsevier, pp. 67-89, 2006.

[12] Harvey R. Brown and James Read, "Clarifying Possible Misconceptions in the Foundations of General Relativity", American Journal of Physics 84(5), pp. 327-334, 2016.

[13] Harvey R. Brown and Roland Sypel, "On the Meaning of the Relativity Principle and Other Symmetries", International Studies in the Philosophy of Science 9(3), pp. 235-253, 1995.

[14] Sean Carroll and Eugene Lim, "Lorentz-Violating Vector Fields Slow the Universe Down", Physical Review D 70, 123525, 2004. 
[15] John Earman, World Enough and Space-Time: Absolute Versus Relational Theories of Space and Time, Cambridge, MA: MIT Press, 1989.

[16] Albert Einstein, "Le principe de relativité et ses conséquences dans la physique moderne", Archives des Sciences Physiques et Naturelles, 29, 5-28, 1910.; republished in The Collected Papers of Albert Einstein, Vol. 3, The Swiss Years: Writings 1909-1911, M. J. Klein, A. J. Kox, J. Renn, and R. Schumann (eds.), Princeton University Press, Princeton 1993.

[17] Albert Einstein, Ideas and Opinions, New York: Bonanza, 1954.

[18] Michael Friedman, Foundations of Space-Time Theories, Princeton: Princeton University Press, 1983.

[19] J. Frohlich, "Spin, or Actually: Spin and Quantum Statistics", in B. Duplantier, J.-M. Raimond and V. Rivasseau (eds.), The Spin, Basel: Birkhäuser Verlag, pp. 1-60, 2009.

[20] Nick Huggett, "Reflections on Parity Nonconservation", Philosophy of Science 67(2), pp. 219-241, 2000.

[21] Ted Jacobson and David Mattingly, "Gravity with a Dynamical Preferred Frame", Physical Review D 64, 024028, 2001.

[22] Michel Janssen, "COI Stories: Explanation and Evidence in the History of Science", Perspectives on Science 10(4), pp. 457-522, 2002.

[23] Michel Janssen, "Drawing the Line Between Kinematics and Dynamics in Special Relativity", Studies in History and Philosophy of Modern Physics 40, pp. 26-52, 2009.

[24] Eleanor Knox, "Newton-Cartan Theory and Teleparallel Gravity: The Force of a Formulation", Studies in the History and Philosophy of Modern Physics 42, pp. 264-275, 2011.

[25] Eleanor Knox, "Newtonian Spacetime Structure in Light of the Equivalence Principle", British Journal for the Philosophy of Science 65(4), pp. 863-880, 2014.

[26] Eleanor Knox, "Physical Relativity from a Functionalist Perspective", Studies in History and Philosophy of Modern Physics, 2017. (Forthcoming.) 
[27] E. Kretschmann, "Über den physikalischen Sinn der Relativitätspostulate", Annalen der Physik 53, pp. 575-614, 1917.

[28] Marc Lange, "Laws and Meta-Laws of Nature: Conservation Laws and Symmetries", Studies in History and Philosophy of Modern Physics 38, pp. 457-481, 2007.

[29] J. M. Leinaas and J. Myrheim, "On the theory of identical particles", Il Nuovo Cimento B 37(1), pp. 1-23, 1977.

[30] David Malament, Topics in the Foundations of General Relativity and Newtonian Gravitation Theory, Chicago: University of Chicago Press, 2012.

[31] Tim Maudlin, Philosophy of Physics: Space and Time. Princeton, NJ: Princeton University Press, 2012.

[32] D. J. Miller, "A Constructive Approach to the Special Theory of Relativity," American Journal of Physics 78, pp. 633-638, 2010.

[33] Wayne C. Myrvold, "How Could Relativity be Anything Other Than Physical?", Studies in History and Philosophy of Modern Physics, 2017. (Forthcoming.)

[34] W. M. Nelson, Relativity Made Real: Under the Hood of Einstein's Theory, second edition, Charleston, SC: CreateSpace Publishing, 2013.

[35] W. M. Nelson, "Special Relativity from the Dynamical Viewpoint", American Journal of Physics 83(7), pp. 600-607, 2015.

[36] Oliver Passon, "What you always wanted to know about Bohmian mechanics but were afraid to ask", http://philsciarchive.pitt.edu/3026/1/bohm.pdf

[37] J. Brian Pitts, "Absolute Objects and Counterexamples: Jones-Geroch Dust, Torretti Constant Curvature, Tetrad-Spinor, and Scalar Density", Studies in History and Philosophy of Modern Physics 37(2), pp. 347-371, 2006.

[38] J. Brian Pitts, "Space-Time Constructivism vs. Modal Provincialism: Or, How Special Relativistic Theories Needn't Show Minkowski Chronogeometry", Studies in History and Philosophy of Modern Physics, 2017. (Forthcoming.) 
[39] Oliver Pooley, "Substantivalist and Relationist Approaches to Spacetime", in R. Batterman (ed.), The Oxford Handbook of Philosophy of Physics, Oxford University Press, 2013.

[40] Oliver Pooley, "Background Independence, Diffeomorphism Invariance, and the Meaning of Coordinates", in Dennis Lehmkuhl, Gregor Schiemann and Erhard Scholz (eds.), Towards a Theory of Spacetime Theories, Birkhäuser, 2017.

[41] R. E. Prange and S. M. Girvin (eds.), The Quantum Hall Effect, Graduate Texts in Contemporary Physics, Springer-Verlag, 1990.

[42] James Read, "Explanation, Geometry, and Conspiracy in Relativity Theory", 2017.

[43] James Read, Harvey R. Brown and Dennis Lehmkuhl, "Two Miracles of General Relativity", Studies in History and Philosophy of Modern Physics, 2018. (Forthcoming.)

[44] Ward Struyve "Pilot-wave approaches to quantum field theory", https://arXiv:1101.5819v1 [quant-ph]

[45] Roland Sypel and Harvey R. Brown, "When is a Physical Theory Relativistic?", in D. Hull, M. Forbes and K. Okruhlik (eds.), Proceedings of the 1992 Biennial Meeting of the Philosophy of Science Association, volume 1, Philosophy of Science Association, East Lansing, Michigan, pp. 507-514, 1992.

[46] Robert Wald, General Relativity, Chicago: University of Chicago Press, 1984.

[47] David Wallace, "Who's Afraid of Coordinate Systems? An Essay on Representation of Spacetime Structure", Studies in History and Philosophy of Modern Physics, 2017. (Forthcoming.)

[48] James Owen Weatherall, "On (Some) Explanations in Physics", Philosophy of Science 78(3), pp. 421-447, 2011.

[49] James Owen Weatherall, "Conservation, Inertia, and Spacetime Geometry", Studies in History and Philosophy of Modern Physics, 2017. (Forthcoming.) 